\title{
PROGRESS IN WIDE FIELD CCD ASTROMETRY
}

\author{
N. ZACHARIAS \\ USRA/USNO Washington DC, USA
}

\section{Astrometric Performances of 5 Telescopes}

Instrumental parameters and astrometric results from five telescopes are summarized in Table 1. The KPNO $0.9 \mathrm{~m}$ has field-corrector optics. The CTIO $0.9 \mathrm{~m}$ is a classical Cassegrain. The 4 -meter telescopes both have doublet field-correctors. The USNO $0.2 \mathrm{~m}$ (8-inch) astrograph has a 5-element lens which is designed for a $9^{\circ}$ flat field of view for photographic plates. A bandpass of $570-650 \mathrm{~nm}$ is used at the USNO $0.2 \mathrm{~m}$ while most frames with the other telescopes have been taken through a Gunn $r(600-710 \mathrm{~nm})$ filter. Stellar images on the CCD frames have been fitted with a 2-D circular symmetric Gaussian profile, giving the centroiding error $\sigma_{f i t}$. Plots of $\sigma_{f i t}$ vs. instrumental magnitude look similar in shape for all telesopes. The asymptotic fit precision, $\sigma_{a f p}$, is the limit in $\sigma_{f i t}$ achieved for bright stars, given in milli pixels (mpx) in Table 1. This centering error is overestimated by an amount depending on the deviation of the real image profile from the model function (Winter 1997).

A measure of the repeatability of the observations, $\sigma_{f f}$, has been obtained from frame to frame comparisons of centrally overlapping frames using a linear transformation model. The error $\sigma_{a t m}$ due to the turbulence in the atmosphere accounts for about $50 \%$ of $\sigma_{f f}$ for $\approx 200 \mathrm{sec}$ exposure times (Zacharias 1996).

For the reflecting telescopes in this investigation, a significant part of the general field distortion pattern is the third order optical distortion (D3) term. D3, $\sigma_{D 3}$ and the center of distortion have been determined by $x, y$ transformations of frames overlapping by about $50 \%$ in area (Zacharias et al. 1995). A significant offset of the center of the D3 term with respect to the center of the CCD has been found for each of the $0.9 \mathrm{~m}$ telescopes, which varies from one observing run to another. The small and constant 
TABLE 1. Telescope parameters and astrometric results

\begin{tabular}{lrrrrrrrr}
\hline telescope & $\begin{array}{r}\text { pixel } \\
\mu m\end{array}$ & $\begin{array}{r}\text { scale } \\
\text { "/px }\end{array}$ & $\begin{array}{r}\text { FOV } \\
\text { arcmin }\end{array}$ & $\begin{array}{r}\sigma_{a f p} \\
\mathrm{mpx}\end{array}$ & $\begin{array}{r}\sigma_{f f} \\
\mathrm{mpx}\end{array}$ & $\begin{array}{r}\sigma_{f f} \\
\mathrm{mas}\end{array}$ & $\begin{array}{r}\mathrm{D} 3 \\
\mathrm{px} / \mathrm{px}^{3}\end{array}$ & $\begin{array}{r}\sigma_{D 3} \\
\mathrm{px} / \mathrm{px}^{3}\end{array}$ \\
\hline CTIO 4m & 24 & 0.43 & $15 \times 15$ & 12 & 13 & 6 & $-1.53 \mathrm{e}-9$ & $0.02 \mathrm{e}-9$ \\
KPNO 4m & 24 & 0.47 & $16 \times 16$ & 13 & 15 & 7 & $-2.07 \mathrm{e}-9$ & $0.01 \mathrm{e}-9$ \\
\hline CTIO .9m & 24 & 0.40 & $13 \times 13$ & 16 & 15 & 6 & $-0.45 \mathrm{e}-9$ & $0.03 \mathrm{e}-9$ \\
KPNO .9m & 24 & 0.68 & $23 \times 23$ & 12 & 11 & 7 & $-0.49 \mathrm{e}-9$ & $0.02 \mathrm{e}-9$ \\
\hline USNO .2m & 9 & 0.90 & $23 \times 15$ & 12 & 11 & 10 & $2.2 \mathrm{e}-13$ & $<0.2 \mathrm{e}-13$ \\
\hline
\end{tabular}

TABLE 2. Characteristics of a new astrometric survey

\begin{tabular}{lrl}
\hline CCD detector & $4 \mathbf{k} \times 4 \mathbf{k}$ & KODAK \\
readout noise & 15 & $e^{-} /$pixel \\
field of view & $60 \times 60$ & arcmin \\
exposure time & 120 & seconds, guided \\
observing throughput & 15 & frames/hour \\
req. observing time & 3500 & hours/hemisphere, 2-fold \\
& $\leq 2$ & year at a good site \\
\hline estimated catalog accuracy & 20 & mas, $R=6 \ldots 13.5$ mag \\
& 30 & mas, $R=15.0$ mag \\
& 70 & mas, $R=16.0$ mag \\
average & 2000 & stars / frame \\
total & 40 & million stars / hemisphere \\
long exposure access & $\geq 100$ & RORF sources/hemisphere \\
\hline
\end{tabular}

D3 term for the USNO astrograph has been determined from external plate solutions using a 5 degree field.

The mean FWHM, mean image elongation and $\sigma_{a f p}$ are important parameters for assessing the astrometric quality of the CCD observations. The USNO astrograph is more diffraction than seeing limited. Thus larger than average FWHMs indicate a focus setting problem while larger than average mean image elongations indicate a guiding problem. For the KPNO and CTIO $0.9 \mathrm{~m}$ telescopes the mean image elongation is strongly correlated with focus setting because of astigmatism present at the field edges, particularly at the CTIO $0.9 \mathrm{~m}$. A large FWHM with these and the 4-meter telescopes is an indication for poor seeing. 


\section{High Precision Astrometric Catalog}

Planning has begun at the USNO for a global, high precision, astrometric sky survey using the $0.2 \mathrm{~m}$ astrograph equipped with a CCD camera (Table 2) (Gauss et al. 1996). Current studies (Zacharias \& Rafferty 1996, Zacharias 1997) show that the projected accuracy, which includes an estimate of the systematic errors, is achievable.

\section{Astrometric Calibration Fields}

Following is a recommendation for standard fields to be used for astrometric calibrations at current epochs, selected from the radio-optical reference frame (RORF) list. Most fields are close to the galactic plane (small b). Coordinates of the field centers are for J2000. A status flag (f) indicates particularly good $(\mathrm{g})$ or poor $(\mathrm{p})$ observational coverage as of today.

\begin{tabular}{|c|c|c|c|c|c|c|c|c|}
\hline north & & & equatos & & & south & & \\
\hline $\mathrm{h} \mathrm{m} \mathrm{s}$ & $d, "$ & $\mathrm{~b}$ & $\mathrm{~h} \mathrm{~ms}$ & $d$, " & $b f$ & $\mathrm{~h} \mathrm{~m} \mathrm{~s}$ & d，" & $b f$ \\
\hline 010246 & +582411 & 5 & 033931 & -014636 & 43 & 064814 & -304420 & 14 \\
\hline 023752 & +284809 & 29 & 050113 & -015914 & 25 & 092752 & -203451 & 21 \\
\hline 064632 & +445117 & 18 & 074554 & -004418 & 12 & 111827 & -463415 & 13 \\
\hline 095457 & +174331 & 48 & 090910 & +012136 & 31 & 142756 & -420619 & 17 \\
\hline 183250 & +283338 & 17 & 165833 & +051516 & 27 & 170053 & -261052 & $10 \mathrm{p}$ \\
\hline 211529 & +293338 & 13 & 210139 & +034131 & 27 & 191110 & -200655 & $13 \mathrm{p}$ \\
\hline 220315 & +314538 & 19 & 225718 & +024318 & 49 & & & \\
\hline
\end{tabular}

\section{References}

Gauss,F.S., Zacharias,N., Rafferty,T.J., Germain,M.E., Holdenried,E.R., Pohlman,J. and Zacharias,M.I. 1996, Bull.Am.Astron.Soc. in preparation

Winter,L. 1997, diss. Univ.of Hamburg, in preparation

Zacharias,N. de Vegt,C. Winter,L. Johnston,K. 1995, Astron.J., 110, 3093

Zacharias,N., Rafferty,T.J. 1995, Bull.Am.Astron.Soc,, 27, 1302

Zacharias,N., 1996, in press Publ.Astron.Soc.Pacific, December issue

Zacharias,N., 1997, in prep. for Astron.J. 